\title{
PÚRPURA TROMBOCITOPÉNICA IDIOPATICA EN ADULTOS: EXPERIENCIA EN UN HOSPITAL DE TERCER NIVEL, LA PAZ, BOLIVIA
}

\author{
IDIOPATHIC THROMBOCYTOPENIC PURPURA IN ADULTS: EXPERIENCE \\ FROM A TERTIARY CARE HOSPITAL, LA PAZ, BOLIVIA
}

\author{
Nelson Miguel Nina García ${ }^{1}$, Hernán Huarita ${ }^{2}$
}

\section{RESUMEN}

Introducción: La purpura trombocitopénica idiopática es un trastorno adquirido causado por auto-anticuerpos contra antígenos plaquetarios. A la fecha no se han estudiado las características clínicas y respuesta al tratamiento en Bolivia. Objetivo: Describir las características clínicas y resultados del tratamiento en pacientes adultos con purpura trombocitopénica idiopática de reciente diagnóstico. Métodos: Se realizó un estudio descriptivo, retrospectivo de una serie de casos de adultos con púrpura trombocitopénica idiopática en un Servicio de Hematología de La Paz, Bolivia durante el periodo 2009-20I4. Resultados: El análisis incluyó 23 pacientes con una edad media de 59,9 años. Al diagnóstico todos los pacientes presentaron manifestaciones hemorrágicas (nasal $82,6 \%$, cutáneo $56,5 \%$, y oral 34,78\%), la media del recuento plaquetario al diagnóstico fue de $26.7 \times 109 / \mathrm{L}$. Se detectó anticuerpos contra Helicobacter pylori en 4 de 6 que contaban con esta prueba. El hallazgo más frecuente en médula ósea fue la presencia de un número aumentado o normal de megacariocitos. La respuesta al tratamiento de primera línea fue del 96\% (RC: 61\%, R: 35\%). Tras una media de seguimiento de 24 meses, el $60 \%$ de los pacientes presentaron recaída en un promedio de 12,5 meses desde el diagnóstico. Conclusiones: Al momento del diagnóstico las manifestaciones hemorrágicas son las más frecuentes, los recuentos plaquetarios por lo general son inferiores a 30 × 109/L. Los corticoides son el tratamiento inicial de elección, con respuestas favorables en la mayoría de los pacientes, sin embargo, más de la mitad de los pacientes presentan recaída en tiempo promedio de I año.

\section{ABSTRACT}

Introduction: Idiopathic thrombocytopenic purpura is an acquired disorder caused by autoantibodies against platelet antigens. To date have not been studied the clinical characteristics and treatment response in Bolivia. Objective: To describe the clinical characteristics and treatment outcomes in adult patients with newly diagnosed immune thrombocytopenic purpura.Methods:A descriptive, retrospective study of an adult case series with immune thrombocytopenic purpura was performed in a Hematology Service of La Paz, Bolivia during the period 2009-2014. Results: We included 23 patients with a median age of 59.9 years.All patients had hemorrhagic manifestations at diagnosis (nasal $82.6 \%$, oral $56.5 \%$ and cutaneous 34.78\%). The average platelet count was 26.7 x 109/L. The presence of antibodies against Helicobacter pylori was detected in 4 of 6 patients. The presence of normal or increased megakaryocyte numbers was the predominant finding in bone marrow.

The response to first-line treatment was $96 \%$ (CR: 6I\%, R: 35\%). After a mean follow-up of 24 months, $60 \%$ of the patients had a relapse in an average of 12.5 months from diagnosis. Conclusions: At the time of diagnosis, hemorrhagic manifestations are the most frequent, platelet counts are usually less than $30 \times 109 / \mathrm{L}$. Corticosteroids are the initial treatment of choice, with favorable responses in the majority of patients, however, more than half of the patients present relapse in an average time of I year.

\section{INTRODUCCIÓN}

T a purpura trombocitopénica idiopática (PTI)

Ltambién llamada púrpura trombocitopénica inmune, es un trastorno adquirido caracterizado por la presencia de auto-anticuerpos contra antígenos plaquetarios $^{1}$. Puede ser catalogada como primaria o secundaria en función a la ausencia o presencia de un trastorno subyacente. Según la literatura actual se define PTI primaria como La purpura trombocitopénica idiopática (PTI) también llamada púrpura trombocitopénica inmune, es un trastorno adquirido caracterizado por la presencia de autoanticuerpos contra antígenos plaquetarios ${ }^{1}$. Puede ser catalogada como primaria o secundaria en función a la ausencia o presencia de un trastorno subyacente.
1 Especialista en Hematología - Hemoterapia, Servicio de Hematología, Hospital Materno Infantil,

La Paz, Bolivia

${ }^{2}$ Médico Patólogo Clínico, Hospital Obrero No 1, La Paz, Bolivia Correspondencia a:

Nombre: Dr. Nelson Miguel Nina García

Correo electrónico: nelson_ninag@outlook.com

Telf. y celular: 72472559

Palabras clave: Purpura trombocitopénica idiopática, signos y síntomas, Bolivia, helicobacter pylori, trombocitopenia

Keywords: Idiopatic thrombocytopenic purpura, signs and symptoms, Bolivia, helicobacter pylori, thrombocytopenia

Procedencia y arbitraje: no comisionado, sometido a arbitraje externo.

Recibido para publicación: 20 de octubre de 2018 Aceptado para publicación: 31 de agosto de 2019

Citar como:

Nina N, Huarita H. Púrpura trombocitopenica idiopatica en adultos: experiencia en un hospital de tercer nivel, La Paz, Bolivia. Rev Cient Cienc Med 2018;21(2): 7-14 
Según la literatura actual se define PTI primaria como la presencia de trombocitopenia aislada (recuento de plaquetas $<100 \times 109 / \mathrm{L}$ ) en ausencia de otras causas o trastornos que pueden estar asociados con trombocitopenia, en cambio la PTI secundaria se define como cualquier forma de PTI distinta a la primaria; esta incluye aquellas que son secundarias a enfermedades autoinmunes, infecciones y trastornos linfoproliferativos ${ }^{1,2}$.

La patogénesis de la PTI no está del todo comprendida, contribuyen tanto la reducción del tiempo de vida plaquetaria debido a la destrucción mediada por anticuerpos, así como una producción disminuida de plaquetas ${ }^{2}$. El mecanismo subyacente involucra auto-anticuerpos tipo IgG específicos que son producidos por los linfocitos B del paciente, generalmente dirigidos contra las glicoproteínas de la membrana plaquetaria como la glicoproteína (GP) $\mathrm{IIb} / \mathrm{III}$. Los eventos que desencadenan mecanismos específicos no están claros ${ }^{3}$.

Es un trastorno relativamente raro con incidencias anuales de 1,6 cada 100.000 adultos en los Estados Unidos y de 2,68 por cada 100.000 adultos en Europa ${ }^{4}$. Los datos acerca de la incidencia de este trastorno en Bolivia aún se desconocen.

La PTI tiene un curso clínico altamente variable en cuanto a duración y severidad, además la respuesta a los tratamientos convencionales no es predecible. Los tratamientos se dividen en aquellos de primera línea conformados por corticoides, inmunoglobulina intravenosa (Ig IV) e inmunoglobulina anti-D, y los de segunda línea conformados por esplenectomía y otros agentes terapéuticos ${ }^{5}$.

Realizarun estudioepidemiológicoes relativamente difícil debido a los múltiples mecanismos patogénicos y la no presencia de hallazgos específicos. De esta forma el presente estudio describe una serie de casos de PTI en Bolivia, abordándose los aspectos clínicos, laboratoriales y terapéuticos.

\section{MATERIALES Y MÉTODOS}

Se realizó un estudio descriptivo, retrospectivo de una serie de casos de PTI de reciente diagnóstico, en el servicio de Hematología del Hospital Materno Infantil de la Caja Nacional de Salud, La Paz, Bolivia, durante el periodo 2009-2013.

El universo del estudio estuvo constituido por todos los pacientes diagnosticados con PTI durante el periodo de estudio, siendo un total de 60 casos según registro interno de la institución. La población de estudio la conformaron todos los pacientes que cumplían los siguientes criterios de inclusión: pacientes con edad igual o mayor a 18 años que cumplían con los criterios para la definición de caso de PTI ${ }^{5}$, pacientes que fueron diagnosticados en el Servicio de Hematología del Hospital Materno Infantil y que contaban con seguimiento hasta la fecha de recolección de datos.

Luego de proceder a la revisión de historiales clínicos y la valoración de los criterios de inclusión, de los 60 casos inicialmente identificados en el registro, fueron excluidos 37 casos: 33 casos debido a que no continuaron el seguimiento después del diagnóstico inicial o el historial clínico no estaba disponible, 4 casos debido a que no fueron diagnosticados en la institución donde se efectuó el estudio. Un total de 23 pacientes fueron incluidos para el análisis de datos.

La recolección de datos se efectuó desde septiembre de 2014 a febrero de 2015. Los datos registrados fueron: edad, sexo, manifestaciones clínicas, comorbilidades, recuentos plaquetarios, exámenes adicionales (perfil inmunológico, anticuerpos anti-plaquetarios, serologías virales, serología para Helicobacter pylori, aspirado de medula ósea), el tratamiento inicial, respuesta terapéutica y duración de la misma. La respuesta al tratamiento fue valorada según criterios establecidos: respuesta completa ( $\mathrm{RC}$ ) en presencia de recuento plaquetario $>100$ x 109/L y ausencia de sangrado; respuesta (R) en presencia de recuento plaquetario $\geq 30 \mathrm{x}$ 109/L o un incremento de más de 2 veces la cifra basal y ausencia de sangrado; no respuesta (NR) en presencia de recuento plaquetario < $30 \times 109 / \mathrm{L}$ o incremento menor de 2 veces la cifra basal o presencia de síntomas hemorrágicos6.

Los datos fueron procesados en el programa Epidat versión 4.0 para la obtención de frecuencias (relativas y absolutas), media y desviación estándar.

\section{RESULTADOS}

\section{Características Generales}

La media de edad de los pacientes del estudio fue 59,91 años (rango 27-85 años), 14 pacientes del sexo femenino $(60,86 \%)$ y 9 pacientes del sexo masculino $(39,13 \%)$.

13 pacientes $(56,5 \%)$ presentaban comorbilidades asociadas, por orden de frecuencia: enfermedades cardiovasculares (principalmente hipertensión arterial sistémica), seguidas de las endocrinológicas (principalmente diabetes mellitus tipo 2), genitourinarias (hiperplasia prostática benigna) y antecedentes de patología neoplásica (Tabla 1). Dos pacientes presentaron más de una comorbilidad.

\section{Manifestaciones Hemorrágicas}


Todos los pacientes presentaron alguna manifestación hemorrágica al momento del diagnóstico. Según sitio anatómico, las manifestaciones hemorrágicas a nivel nasal y cutáneo fueron las más frecuentes con un $82,60 \%$ y $56,50 \%$ respectivamente, otros sitios implicados con menos frecuencia fueron la cavidad oral, aparato genitourinario y tracto gastrointestinal (Figura 1).

\section{Hallazgos de laboratorio}

El recuento plaquetario al diagnóstico fue en promedio de 26,7 x 109/L (rango 5,0 a 62,0 x $109 / \mathrm{L})$. Un $47 \%$ de los pacientes tenían un recuento plaquetario igual o menor a 20,0 x109/L.

Se realizaron otras pruebas diagnósticas en 14 pacientes, como la determinación de anticuerpos antiplaquetarios, serologías virales, perfil inmunológico, serología para Helicobacter pylori y aspirado de médula ósea. La determinación de anticuerpos antiplaquetarios se efectuó en 7 pacientes (30\%) con títulos positivos en todos ellos. En relación al perfil inmunológico se valoró la determinación por ELISA de anticuerpos antinucleares (ANA) $\mathrm{y}$ anti-DNAds los cuales fueron solicitados en 14 pacientes, se evidenció títulos positivos para ANA en 3 pacientes $(21 \%)$ y anti-DNAds en 1 paciente $(7 \%)$.

En un grupo similar de 14 pacientes se realizó determinación serológica por ELISA para el virus de inmunodeficiencia humana (VIH), virus de la hepatitis C (VHC) y complejo TORCH (Tabla 2). No se encontró positividad para VIH y VHC, un caso presentó anticuerpos del tipo $\operatorname{IgM}$ para citomegalovirus (7\%). Se efectuó la detección de anticuerpos contra Helicobacter pyloripor técnicas serológicas en 6 pacientes siendo positivo en 4 de ellos.

El aspirado y frotis de medula ósea se realizó en 13 pacientes, los principales hallazgos se muestran en la tabla 2.

\section{Aspectos terapéuticos}

Los corticoides fueron el tratamiento de primera línea en todos los pacientes, en monoterapia o en asociación con Ig IV. La monoterapia con corticoides se efectuó en 16 pacientes (10 recibieron dexametasona y 6 recibieron prednisona). En los casos restantes ( 7 pacientes) al tratamiento se asoció Ig IV (Tabla 3).

\section{Figura 1. Manifestaciones hemorrágicas según sitio anatómico (expresado en porcentaje)}

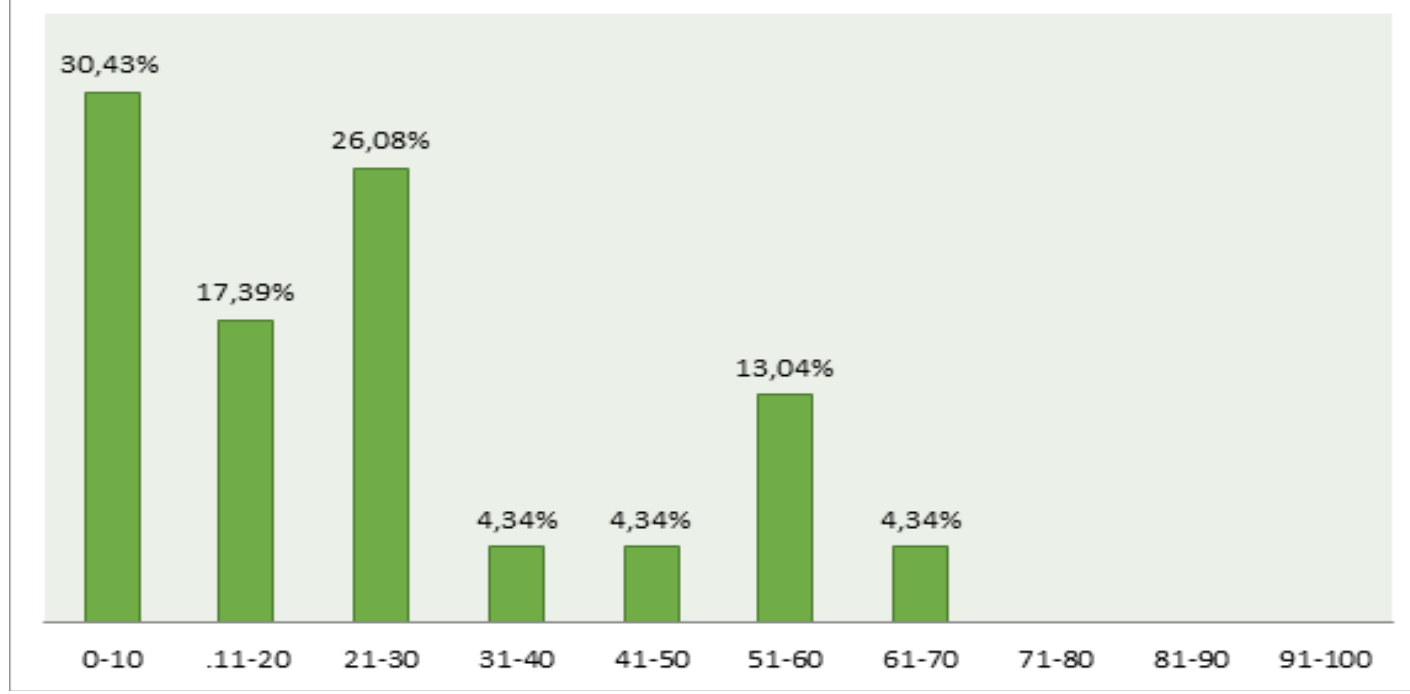

Figura 2. Recuento Plaquetario al diagnóstico (x 109/L)

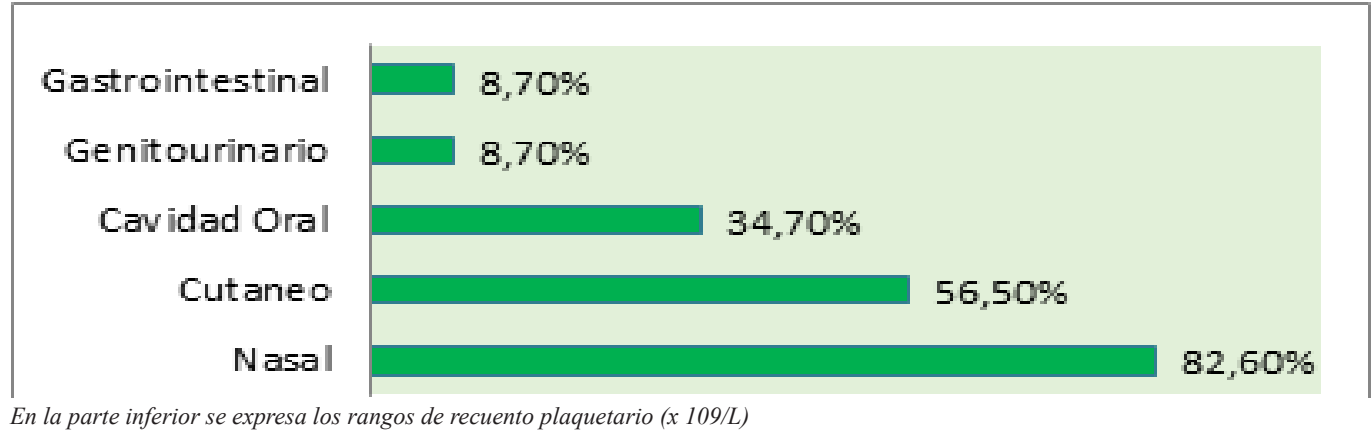

En la parte inferior se expresa los rangos de recuento plaquetario (x 109/L) 
Tabla 1. Características generales del grupo de estudio

\begin{tabular}{|lc|}
\hline \multicolumn{1}{c}{ Variable } & Media (rango) \\
\hline Media de edad en años de todo el grupo de estudio (rango) & 59,91 años (27-85) \\
\hline Media de edad en años para el sexo masculino (rango) & $\mathbf{5 6 , 5}$ años (27-74) \\
\hline Pacientes de sexo masculino (\%) & 65,22 años (46-85) \\
\hline Pacientes de sexo femenino (\%) & Frecuencia n (\%) \\
\hline Comorbilidades (\%) & $9(39,13)$ \\
\hline Total del grupo de estudio & $14(60,86)$ \\
\hline Enfermedades cardiovasculares & \\
\hline Enfermedades endocrinológicas & $13(56,52)$ \\
\hline Enfermedades urológicas & $5(21,73)$ \\
\hline Antecedente de neoplasias & $4(17,39)$ \\
Enfermedades inmunológicas & $3(13,04)$ \\
\hline Enfermedades dermatológicas & $2(8,69)$ \\
\hline
\end{tabular}

Tabla 2. Resultados de otros estudios laboratoriales al momento del diagnóstico (\%)

\begin{tabular}{lll}
\hline Prueba (Número de pacientes en los que se efectuó la prueba) & Positivo (\%) & Negativo $(\%)$ \\
\hline Anticuerpos antiplaquetarios $(\mathrm{n}=7)$ & $7(100)$ & $0(0 \%)$ \\
\hline Anticuerpos antinucleares $(\mathrm{n}=14)$ & $3(21)$ & $11(79)$ \\
\hline Anti DNAds $(\mathrm{n}=14)$ & $1(7)$ & $13(93)$ \\
VIH $(\mathrm{n}=14)$ & $0(0)$ & $14(100)$ \\
Hepatitis C $(\mathrm{n}=14)$ & $0(0)$ & $14(100)$ \\
\hline Helicobacter pylori $(\mathrm{n}=6)$ & $4(67)$ & $2(33)$ \\
Hallazgos en el frotis de medula ósea & & Frecuencia $(\%)$ \\
Hiperplasia megacariocítica con megacariocitos basófilos & & $9(69,23)$ \\
Número y morfología adecuada de megacariocitos & & $2(15,38)$ \\
Otros hallazgos & & $2(15,38)$
\end{tabular}

Tabla 3. Resultados del Tratamiento de Primera línea

\begin{tabular}{cccccc} 
Tratamiento & $\mathbf{N}^{\circ}$ pacientes & RC $(\%)$ & R $(\%)$ & NR $(\%)$ & Recaída $(\%)$ \\
\hline Dexametasona & 10 & $7(70)$ & $3(30)$ & 0 & $6(60)$ \\
Prednisona & 6 & $4(67)$ & $2(33)$ & 0 & $6(100)$ \\
Ig IV+ Corticoides & 7 & $3(43)$ & $3(43)$ & $1(14)$ & $2(29)$
\end{tabular}

La respuesta al tratamiento fue valorada en todos un $82,60 \%$ y $56,50 \%$ respectivamente, otros sitios implicados con menos frecuencia fueron la cavidad oral, aparato genitourinario y tracto gastrointestinal (Figura 1).

\section{Hallazgos de laboratorio}

El recuento plaquetario al diagnóstico fue en promedio de 26,7 x 109/L (rango 5,0 a 62,0 x $109 / \mathrm{L})$. Un $47 \%$ de los pacientes tenían un recuento plaquetario igual o menor a 20,0 x109/L.

Se realizaron otras pruebas diagnósticas en 14 pacientes, como la determinación de anticuerpos antiplaquetarios, serologías virales, perfil inmunológico, serología para Helicobacter pylori y aspirado de médula ósea. La determinación de anticuerpos antiplaquetarios se efectuó en 7 pacientes (30\%) con títulos positivos en todos ellos. En relación al perfil inmunológico se valoró la determinación 
por ELISA de anticuerpos antinucleares (ANA) $\mathrm{y}$ anti-DNAds los cuales fueron solicitados en 14 pacientes, se evidenció títulos positivos para ANA en 3 pacientes $(21 \%)$ y anti-DNAds en 1 paciente $(7 \%)$.

En un grupo similar de 14 pacientes se realizó determinación serológica por ELISA para el virus de inmunodeficiencia humana $(\mathrm{VIH})$, virus de la hepatitis C (VHC) y complejo TORCH (Tabla $2)$. No se encontró positividad para $\mathrm{VIH}$ y VHC, un caso presentó anticuerpos del tipo $\operatorname{IgM}$ para citomegalovirus (7\%). Se efectuó la detección de anticuerpos contra Helicobacter pyloripor técnicas serológicas en 6 pacientes siendo positivo en 4 de ellos.

El aspirado y frotis de medula ósea se realizó en 13 pacientes, los principales hallazgos se muestran en la tabla 2.

\section{Aspectos terapéuticos}

Los corticoides fueron el tratamiento de primera línea en todos los pacientes, en monoterapia o en asociación con Ig IV. La monoterapia con corticoides se efectuó en 16 pacientes (10 recibieron dexametasona y 6 recibieron prednisona). En los casos restantes ( 7 pacientes) al tratamiento se asoció Ig IV (Tabla 3).

La respuesta al tratamiento fue valorada en todos los pacientes, 14 pacientes (61\%) presentaron respuesta completa, 8 pacientes $(35 \%)$ presentaron respuesta, 1 paciente no presentó respuesta.

Con tiempo medio de seguimiento de 26,68 meses (1-102 meses), un 60\% de los pacientes presentaron recaída al tratamiento inicial. El tiempo promedio entre el diagnóstico y la recaída fue de 12,58 meses (rango 1-64 meses).

\section{DISCUSIÓN}

Este es el primer estudio realizado en Bolivia que analiza los aspectos clínicos y terapéuticos de la PTI recién diagnosticada en la población adulta. Todos los pacientes de nuestra serie de casos presentaron manifestaciones hemorrágicas al momento del diagnóstico, con mayor frecuencia de origen nasal $(82,6 \%)$, seguidas por las de origen cutáneo y cavidad oral. La literatura indica que la hemorragia es la manifestación clínica más común de la PTI, y generalmente se presenta como hemorragia mucocutánea que afecta a la piel, cavidad oral y el tracto gastrointestinal ${ }^{1}$.

Kühne $\mathrm{T}$ y Cols ${ }^{7}$, en un estudio comparativo de PTI de reciente diagnóstico en niños y adultos encontraron la presencia de sangrado en un 69\% de los casos en adultos. Kurata L y Cols ${ }^{8}$ en 6 845 adultos, encontraron que la manifestación hemorrágica más frecuente era la púrpura con un $62,8 \%$, seguido de las gingivorragias en un 19,9\% y epistaxis en un $10 \%$. Koylu TA y Cols ${ }^{9}$ encontraron datos de sangrado en un 53, $2 \%$ de los pacientes estudiados, las equimosis estaban presentes en un $46,9 \%$, moretones con facilidad en $42,9 \%$, petequias en $37 \%$, gingivorragias en $15,7 \%$, epistaxis en $14,9 \%$, hemorragia genitourinaria en $11,4 \%$ y hemorragia del tracto gastrointestinal en un 5,6\%. Ambos estudios son consistentes en que la manifestación hemorrágica de origen cutáneo y de la cavidad oral son las más frecuentes, como se muestra en nuestra serie de casos. También se destaca la baja frecuencia de epistaxis en la literatura (menor al 15\%) en relación a nuestro estudio 9 . En ambos estudios la frecuencia de hemorragia en el sistema nervioso central fue muy baja, nosotros no encontramos ningún caso.

Es preciso destacar que en el presente estudio, todos los pacientes presentaron síntomas hemorrágicos al diagnóstico, sin embargo cabe resaltar que en los estudios previamente mencionados, también incluyeron pacientes que solo presentaban trombocitopenia sin manifestaciones hemorrágicas ${ }^{7,9}$, esto debido al uso creciente de contadores automáticos con indicación rutinaria en diversas situaciones, aumentando la detección de casos asintomáticos de PTI, algo que no sucede en nuestro medio.

El recuento plaquetario inicial en el presente estudio fue en promedio 26,7 x 109/L, un 47\% tenían recuento plaquetario igual o menor a $20 \times 109 / \mathrm{L}$. En el estudio realizado por Kühne, T y cols7 encontraron un recuento plaquetario promedio de 25,4 x 109/L, un valor muy similar a nuestro hallazgo. Bauer $\mathrm{M}$ y cols $^{10}$, evidenciaron que $66 \%$ presentaron recuentos plaquetarios menores a $\leq 10 \times 109 / 1,24 \%>10$ a $\leq$ 30 x 109/1, y $10 \%>30$ a $\leq 100$ x 109/L. De forma similar, en nuestro estudio hay un gran porcentaje de pacientes con recuentos plaquetarios menores a $20 \mathrm{x}$ 109/1 al momento del diagnóstico.

En los pacientes en los que se realizó la determinación de anticuerpos plaquetarios, todos presentaron títulos positivos, en el perfil inmunológico (ANA, anti-DNAds) se observó 3 casos con títulos positivos para ANA y 1 solo caso para anti-DNAds. No se encontró positividad para VIH y VHC, solo un caso mostro positividad inmunológica para citomegalovirus. La presencia de anticuerpos contra Helicobacter pylori estuvo presente en 4 de 6 pacientes que contaban con esta determinación.

En el estudio de Kühne $\mathrm{T}$ y Cols ${ }^{7}$, la presencia de anticuerpos antinucleares (ANA) se encontró en un $10 \%$ de los casos en los que se realizó 
esta determinación. Se evidenció anticuerpos antiplaquetarios en $47 \%$, la presencia de VIH y VHC en 1 y $3 \%$ respectivamente, la presencia de Helicobacter pylori se evidenció en $31 \%$. En nuestro estudio la presencia de Helicobacter pylori se encontró en un $67 \%$ de los que contaban con esta prueba, sin embargo solo se realizó esta determinación en el $26 \%$ de los pacientes estudiados. En el estudio de Kühne $\mathrm{T}$ y Cols $\mathrm{C}^{7}$, esta prueba se realizó en un $50 \%$ de los pacientes.

Si bien es evidente la presencia de infección por Helicobacter pylori en algunos pacientes, sin embargo el porcentaje de pacientes en los que realizó esta prueba es bajo. La PTI y la anemia por deficiencia de hierro inexplicada son las 2 enfermedades extraintestinales asociadas a infección por Helicobacter pylori ${ }^{11}$.La relación entre esta infección y la PTI fue descrita inicialmente en $1998^{12}$, a partir de entonces ha existido resultados contradictorios. Probablemente, el Helicobacter pylori tenga un rol más predominante en países en vías de desarrollo, donde habitualmente hay mayor prevalencia de este microorganismo como sucede en nuestro medio.

Los hallazgos del aspirado de médula ósea en este estudio mostraron que la hiperplasia megacariocítica con megacariocitos basófilos es más frecuente en $69,23 \%$. Según la literatura los hallazgos en la medula ósea son variables, siendo más frecuentes la presencia de un número incrementado (hiperplasia) o normal de megacariocitos y disminución de la ploidia nuclear (hipolobulación) 13,estos hallazgos comparables a los obtenidos en nuestro estudio. Los corticoides fueron la primera línea de tratamiento en todos los pacientes, en monoterapia o en asociación con Ig IV u otros corticoides. Kühne y $\mathrm{Cols}^{7}$ encontraron que de forma general los corticoides son los más usados como tratamiento inicial en pacientes recién diagnosticados con PTI. La mayor parte de los pacientes recibieron solo corticoides y una menor proporción recibió corticoides más Ig IV. Bauer y Cols ${ }^{10}$, encontraron que los corticoides son el tratamiento de elección en la PTI de reciente diagnóstico en un $92 \%$ de los pacientes, seguida de la IgIV en un $50 \%$, estos hallazgos son similares al presente estudio.

En nuestro estudio la respuesta general al tratamiento de primera línea fue de 96 literatura indica que la hemorragia es la manifestación clínica más común de la PTI, y generalmente se presenta como hemorragia mucocutánea que afecta a la piel, cavidad oral y el tracto gastrointestinal ${ }^{1}$.

Kühne $\mathrm{T}$ y Cols ${ }^{7}$, en un estudio comparativo de PTI de reciente diagnóstico en niños y adultos en contraron la presencia de sangrado en un $69 \%$ de los casos en adultos. Kurata L y Cols ${ }^{8}$ en 6845 adultos, encontraron que la manifestación hemorrágica más frecuente era la púrpura con un $62,8 \%$, seguido de las gingivorragias en un $19,9 \%$ y epistaxis en un $10 \%$. Koylu TA y Cols ${ }^{9}$ encontraron datos de sangrado en un 53, $2 \%$ de los pacientes estudiados, las equimosis estaban presentes en un $46,9 \%$, moretones con facilidad en $42,9 \%$, petequias en $37 \%$, gingivorragias en $15,7 \%$, epistaxis en $14,9 \%$, hemorragia genitourinaria en $11,4 \%$ y hemorragia del tracto gastrointestinal en un 5,6\%. Ambos estudios son consistentes en que la manifestación hemorrágica de origen cutáneo y de la cavidad oral son las más frecuentes, como se muestra en nuestra serie de casos. También se destaca la baja frecuencia de epistaxis en la literatura (menor al 15\%) en relación a nuestro estudio 9 . En ambos estudios la frecuencia de hemorragia en el sistema nervioso central fue muy baja, nosotros no encontramos ningún caso.

Es preciso destacar que en el presente estudio, todos los pacientes presentaron síntomas hemorrágicos al diagnóstico, sin embargo cabe resaltar que en los estudios previamente mencionados, también incluyeron pacientes que solo presentaban trombocitopenia sin manifestaciones hemorrágicas ${ }^{7,9}$, esto debido al uso creciente de contadores automáticos con indicación rutinaria en diversas situaciones, aumentando la detección de casos asintomáticos de PTI, algo que no sucede en nuestro medio.

El recuento plaquetario inicial en el presente estudio fue en promedio 26,7 x 109/L, un 47\% tenían recuento plaquetario igual o menor a $20 \times 109 / \mathrm{L}$. En el estudio realizado por Kühne, T y cols7 encontraron un recuento plaquetario promedio de 25,4 x 109/L, un valor muy similar a nuestro hallazgo. Bauer $\mathrm{M} \mathrm{y}$ cols $^{10}$, evidenciaron que $66 \%$ presentaron recuentos plaquetarios menores a $\leq 10 \times 109 / 1,24 \%>10$ a $\leq$ 30 x 109/1, y $10 \%>30$ a $\leq 100$ x 109/L. De forma similar, en nuestro estudio hay un gran porcentaje de pacientes con recuentos plaquetarios menores a $20 \mathrm{x}$ 109/1 al momento del diagnóstico.

En los pacientes en los que se realizó la determinación de anticuerpos plaquetarios, todos presentaron títulos positivos, en el perfil inmunológico (ANA, anti-DNAds) se observó 3 casos con títulos positivos para ANA y 1 solo caso para anti-DNAds. No se encontró positividad para VIH y VHC, solo un caso mostro positividad inmunológica para citomegalovirus. La presencia de anticuerpos contra Helicobacter pylori estuvo presente en 4 de 6 pacientes que contaban con esta determinación.

En el estudio de Kühne $\mathrm{T}$ y $\mathrm{Cols}^{7}$, la presencia 
de anticuerpos antinucleares (ANA) se encontró en un $10 \%$ de los casos en los que se realizó esta determinación. Se evidenció anticuerpos antiplaquetarios en $47 \%$, la presencia de VIH y VHC en 1 y $3 \%$ respectivamente, la presencia de Helicobacter pylori se evidenció en $31 \%$. En nuestro estudio la presencia de Helicobacter pylori se encontró en un $67 \%$ de los que contaban con esta prueba, sin embargo solo se realizó esta determinación en el 26\% de los pacientes estudiados. En el estudio de Kühne $\mathrm{T}$ y Cols ${ }^{7}$, esta prueba se realizó en un $50 \%$ de los pacientes.

Si bien es evidente la presencia de infección por Helicobacter pylori en algunos pacientes, sin embargo el porcentaje de pacientes en los que realizó esta prueba es bajo. La PTI y la anemia por deficiencia de hierro inexplicada son las 2 enfermedades extraintestinales asociadas a infección por Helicobacter pylori ${ }^{11}$.La relación entre esta infección y la PTI fue descrita inicialmente en $1998^{12}$, a partir de entonces ha existido resultados contradictorios. Probablemente, el Helicobacter pylori tenga un rol más predominante en países en vías de desarrollo, donde habitualmente hay mayor prevalencia de este microorganismo como sucede en nuestro medio.

Los hallazgos del aspirado de médula ósea en este estudio mostraron que la hiperplasia megacariocítica con megacariocitos basófilos es más frecuente en $69,23 \%$. Según la literatura los hallazgos en la medula ósea son variables, siendo más frecuentes la presencia de un número incrementado (hiperplasia) o normal de megacariocitos y disminución de la ploidia nuclear (hipolobulación) 13,estos hallazgos comparables a los obtenidos en nuestro estudio. Los corticoides fueron la primera línea de tratamiento en todos los pacientes, en monoterapia o en asociación con Ig IV u otros corticoides. Kühne y Cols ${ }^{7}$ encontraron que de forma general los corticoides son los más usados como tratamiento inicial en pacientes recién diagnosticados con PTI. La mayor parte de los pacientes recibieron solo corticoides y una menor proporción recibió corticoides más Ig IV. Bauer y Cols ${ }^{10}$, encontraron que los corticoides son el tratamiento de elección en la PTI de reciente diagnóstico en un $92 \%$ de los pacientes, seguida de la IgIV en un $50 \%$, estos hallazgos son similares al presente estudio.

En nuestro estudio la respuesta general al tratamiento de primera línea fue de 96\% (RC 61\% y R 35\%). KoyluCols, observaron que un 76,5\% de los pacientes alcanzaron $\mathrm{RC}$ al tratamiento de primera línea ${ }^{9}$. Pamuk y Cols ${ }^{14}$, evidenciaron RC en un 51,9\% de los pacientes que recibieron corticoides como tratamiento de primera línea.

La dexametasona en dosis altas (40 mg cada día por 4 días) produce una mayor proporción de respuesta general $(82,1 \%)$ en comparación con la prednisona (67.4\%). Además la dexametasona se asocia a mayores tasas de respuesta completa en comparación con prednisona $(50.5 \% \text { versus } 26.8 \%)^{15}$.Sin embargo solo alrededor de un tercio de los pacientes mantienen respuestas prolongadas ${ }^{15}$. Estos datos son comparables a los obtenidos en nuestro estudio.

En el presente estudio un total de $60 \%$ de los pacientes evaluados presentaron recaída tras el tratamiento de primera línea. El tiempo promedio entre el diagnóstico y la recaída fue de 12,58 meses después del diagnóstico. Durante el seguimiento Koylu y Cols ${ }^{9}$, encontraron que el $50 \%$ de los pacientes que alcanzaron respuesta al tratamiento de primera línea presentaron recaída en un promedio de 6 meses después del diagnóstico. En el estudio de Pamuk y cols ${ }^{14}$, el 58,2\% de los pacientes que presentaron $\mathrm{RC}$ al tratamiento de primera línea presentaron recaída en un promedio de 33 meses. La variabilidad en cuanto al tiempo de duración de la respuesta es evidente en los diferentes estudios.

$\mathrm{Si}$ bien la información del presente estudio no es generalizable por tratarse de una serie de casos, proporciona un panorama general del comportamiento de este trastorno en Bolivia. Estudios posteriores deberán abordar aspectos inherentes a la epidemiologia, casos sin manifestaciones hemorrágicas, el rol de la infección por Helicobacter pylori y aspectos adicionales relacionados con el tratamiento. 


\section{REFERENCIAS}

1. George JN, Arnold DM. Immune thrombocytopenia (ITP) in adults: Clinical manifestations and diagnosis. En: Uptodate, Leung LL (Ed), Uptodate, Tirnauer JS. [citado 20Jul 2017]. Disponible en :https:// www.uptodate.com/contents/immune-thrombocytopenia-itp-in-adults-clinical-manifestations-and-diagnosis

2. Zufferey A, Kapur R, Semple JW. Pathogenesis and Therapeutic Mechanisms in Immune Thrombocytopenia (ITP). Journal of Clinical Medicine[Internet]. 2017 [citado 20Jul 2017]; 6(2):16. Disponible en: https: //www. mdpi.com/2077-0383/6/2/16

3. Cines DB, Blanchette VS. Immune thrombocytopenic purpura. N Engl J Med[Internet]. 2002 [citado $10 \mathrm{Abr}$ 2017]; 346(13): 995-1008. Disponible en:https://www. nejm.org/doi/full/10.1056/NEJMra010501

4. Fogarty PF, Segal JB. The epidemiology of immune thrombocytopenic purpura. CurrOpinHematol [Internet]. 2007 [citado 10 Abr 2017]; 14(5): 515-519. Disponible en: https://insights.ovid.com/pubmed?pmid=17934361

5. Provan D, Stasi R, Newland AC, Blanchette VS, BoltonMaggs $\mathrm{P}$, Bussel JB, et al. International consensus report on the investigation and management of primary immune thrombocytopenia. Blood [Internet] 2010 [citado 10 Abr 2017]; 115(2): 168-86. Disponible en: http:// www.bloodjournal.org/content/115/2/168.long?sso-checked=true

6. Rodeghiero F, Stasi R, Gernsheimer T, Michel M, Provan D, Arnold DM, et al. Standardization of terminology, definitions and outcome criteria in immune thrombocytopenic purpura of adults and children: report from an international working group. Blood [Internet] 2009 [citado 15 Abr 2017]; 113(11): 2386-93. Disponible en: http://www.bloodjournal.org/content/113/11/2386

7. Kühne T, Berchtold W, Michaels LA, Wu R, Donato H, Espina B, et al. Newly diagnosed immune thrombocytopenia in children and adults: a comparative prospective observational registry of the Intercontinental Cooperative Immune Thrombocytopenia Study Group. Haematologica [Internet] 2011 [citado 15 Abr 2017]; 96(12): 1831-1837.Disponible en: http://www.haematologica. org/content/96/12/1831.long

8. Kurata Y, Fujimura K, Kuwana M, Tomiyama Y, Murata $M$. Epidemiology of primary immune thrombocytopenia in children and adults in Japan: a population-based study and literature review. Int J Hematol[Internet]. 2011[citado 15 Abr 2017]; 93(3):
329-335.Disponible en: https://link.springer.com/article/10.1007\%2Fs12185-011-0791-1

9. Koylu A, Pamuk GE, Uyanik MS, Demir M, Pamuk ON. Immune thrombocytopenia: epidemiological and clinical features of 216 patients in northwestern Turkey. Ann Hematol [Internet]. 2015 [citado 16 Abr 2017]; 94(3): 459-66.Disponible en: https://link.springer.com/ article/ $10.1007 \% 2$ Fs00277-014-2220-z

10. Bauer M, Baumann A, Berger K, Ackermann B, Shlaen R, Schopohl D, Ostermann H. A Retrospective Observational Single-Centre Study on the Burden of Immune Thrombocytopenia (ITP). Onkologie [Internet]2012[citado 16 Abr 2017]; 35(6): 342-348. Disponible en: https://www.karger.com/Article/Abstract $/ 338935$

11. Malfertheiner P, Megraud F, O’Morain C, Bazzoli F, ElOmar E, Graham D, et al. Current concepts in the management of Helicobacter pylori infection: the Maastricht III Consensus Report. Gut [Internet]2007[citado 23 Abr 2017]; 56(6): 772-781. Disponible en: https://gut. bmj.com/content/56/6/772.long

12. Gasbarrini A, Franceschi F, Tartaglione R, Landolfi R, Pola P, Gasbarrini G. Regression of autoimmune thrombocytopenia after eradication of Helicobacter pylori. Lancet [Internet] 1998[citado 10 Abr 2017]; 352(9131): 878. Disponible en: https://www.thelancet.com/journals/ lancet/article/PIIS0140-6736(05)60004-9/fulltext

13. Gunduz E1, Kivanc BK, Arik D, Isiksoy S, Bal C, Akay $\mathrm{OM}$. Bone marrow examination in patients with immune thrombocytopenia: is there anything different in older patients?.Eur J Haematol[Internet]. 2014[citado 10Jun 2017];93(2):157-60. Disponible en: https://onlinelibrary.wiley.com/doi/abs/10.1111/ejh.12320

14. Pamuk GE, Pamuk ON, Başlar Z, Ongören S, Soysal $T$, Ferhanoğlu $B$, et al. Overview of 321 patients with idiopathic thrombocytopenic purpura. Retrospective analysis of the clinical features and response to therapy. Ann Hematol[Internet]. 2002[citado 15 Abr 2017]; 81(8): 436-40. Disponible en: https://link.springer.com/ article/10.1007\%2Fs00277-002-0488-x

15. Wei Y, Ji XB, Wang YW, Wang JX, Yang EQ, Wang $Z C$, et al. High-dose dexamethasone vs prednisone for treatment of adult immune thrombocytopenia: a prospective multicenter randomized trial. Blood[Internet]. 2016[citado 20 Jul 2017]; 127(3):296-302. Disponible en: http://www.bloodjournal.org/content/127/3/296. long?sso-checked=true 